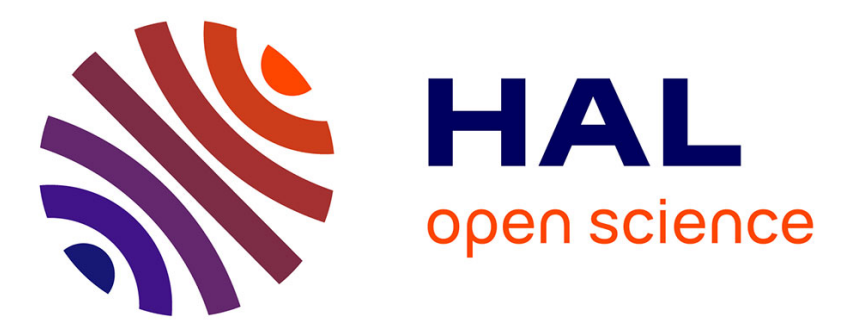

\title{
Computing the elastic properties of sandstone submitted to progressive dissolution
}

\author{
Kajetan Wojtacki, Loïc Daridon, Yann Monerie
}

\section{To cite this version:}

Kajetan Wojtacki, Loïc Daridon, Yann Monerie. Computing the elastic properties of sandstone submitted to progressive dissolution. International Journal of Rock Mechanics and Mining Sciences, 2017, 95, pp.16 - 25. 10.1016/j.ijrmms.2016.12.015 . hal-01502421

\section{HAL Id: hal-01502421 \\ https://hal.science/hal-01502421}

Submitted on 5 Apr 2017

HAL is a multi-disciplinary open access archive for the deposit and dissemination of scientific research documents, whether they are published or not. The documents may come from teaching and research institutions in France or abroad, or from public or private research centers.
L'archive ouverte pluridisciplinaire HAL, est destinée au dépôt et à la diffusion de documents scientifiques de niveau recherche, publiés ou non, émanant des établissements d'enseignement et de recherche français ou étrangers, des laboratoires publics ou privés. 


\title{
Computing the elastic properties of sandstone submitted to progressive dissolution
}

\author{
Kajetan Wojtacki ${ }^{\mathrm{a}}$, Loïc Daridon ${ }^{\mathrm{a}, \mathrm{b}, *}$, Yann Monerie M,b $^{\mathrm{a}}$ \\ ${ }^{a}$ Laboratoire de Mécanique et Génie Civil (LMGC), CNRS UMR 5508, Montpellier, France \\ ${ }^{b}$ Laboratoire de Micromécanique et Intgrité des Structures (MIST), UM, CNRS, IRSN, France
}

\begin{abstract}
We present a numerical method for estimating the stiffness-to-porosity relationships for evolving microstructures of Fontainebleau sandstone. The proposed study is linked to geological storage of $\mathrm{CO}_{2}$ and focuses on long-term and far field conditions, when the progressive degradation of the porous matrix can be assumed to be homogeneous at the sample scale. The method is based on microstructure sampling with respect to morphological descriptors extracted from microtomography. First, an efficient method of generation of accurate numerical media is proposed. The method is based on grain deposit, compaction and diagenesis and allows to reproduce user-defined morphological parameters. Second, two simple numerical models that mimic chemical degradation of porous aquifers are presented. Effective elastic properties are estimated within the framework of periodic homogenization and finite element approach. A fixed-point method on a self-consisted outer layer allows to consider non-periodic representative volume elements. Accurate predictions of elastic properties over a wide range of porosity are obtained. The overall evolutions of elastic behaviour due to the increase of porosity are in excellent agreement both, with experimental data and the results obtained by Arns et al. [1].
\end{abstract}

Keywords: porous media, X-ray microtomography, numerical dissolution, Fontainebleau sandstone, self-consisted numerical method, homogenization

\section{Introduction}

Geological storage of $\mathrm{CO}_{2}$, or Carbon Capture and Storage (CCS), is intensively investigated as a solution to reduce emission of $\mathrm{CO}_{2}$ to the atmosphere. It consists in capturing carbon dioxide at source and then its injection into suitable geological formations [2]. During geological storage, some physical and chemical mechanisms lead to irreversible changes of rock microstructure, and thus of its effective properties. In order to avoid catastrophic damages, it is essential to estimate geological formations behaviour. Effectiveness of geological storage depends on a combination of physical and geochemical trapping phenomena. Carbon dioxide can remain trapped underground by virtue of a number of mechanisms, such as structural and stratigraphic, residual, solubility and mineral trapping [2]. In the pa-

\footnotetext{
*Corresponding author

Email addresses: kajetan.wojtacki@umontpellier.fr (Kajetan Wojtacki), loic.daridon@umontpellier.fr (Loïc Daridon), yann.monerie@umontpellier.fr (Yann Monerie)
}

per we focus on influence of chemical degradations on mechanical behaviour of saline aquifer.

Chemo-mechanical coupling problem has been intensively studied in recent years, following pioneering works on concrete, mortar or cement paste. Obtained results confirm a general material weakening during chemical degradation (loss of strength and stiffness). Experimental studies considering $\mathrm{CO}_{2}$ injection into the rock reservoirs performed in laboratory conditions, relatively close to the injection point, show different behaviours depending on physical parameters of injected fluid (temperature, pressure), chemical composition of injected fluid and initial rock parameters (see Luquot et al. [3], Gouze et al. [4], Eggermann et al. [5], Egermann et al. 2006 [6], Izgec et al. 2008 [7] among others). In experimental research of behaviour of soil at long time scale after $\mathrm{CO}_{2}$ injection (Bemer et al. [8], Nguyen et al. [9]), it is assumed that porous aquifer is saturated by saline water and concentration of dissolved $\mathrm{CO}_{2}$ is uniformly distributed in the porous space. Most of the results showed that chemical degradation can be considered as homogenous at the sample scale. 
Due to increase of porosity, material weakening occurs. Decrease of both, stiffness and shear strength, and increase of permeability have been also noticed. Various approaches are proposed to model this type of chemomechanical coupling. Several analytical models are obtained by periodic homogenization method Peter [10], Meier et al. [11] and Lewandowska [12]. Phenomenological model were also proposed by Coussy [13] in which variations of poroelastic parameters are described as a heuristic function of irreversible porosity caused by chemical degradation. Numerical and computational aspects of chemo-mechanical modelling related to flow and transport phenomena during injection process are presented by Doughty et al. [14], Kumar et al. [15], Hovorka et al. [16] and Wojtacki et al. [17].

The aim of this paper is to estimate evolution of elastic properties of saline aquifer, which is subjected to progressive chemical degradation due to $\mathrm{CO}_{2}$ dissolution and acidification of saline water. The objective is to find a link between evolving material microstructure, phenomena occurring at microscopic scale, and macroscopic behaviour. The proposed study focuses on long-term and far field conditions, when dissolution of porous matrix can be assumed to be homogeneous at sample scale $[6,8]$. Chemical degradation of material is taken into account in a simplified way by performing numerical erosion of the digitized image of the microstructure.

The paper is organised as follows. Section 2 presents a morphological description of the microstructure of Fontainebleau sandstone. In Section 3, numerical samples are generated and the accuracy of their morphology is checked. Section 4 presents two different scenarii for numerical dissolution and a brief summary of morphological descriptors evolution. Finally, in Section 5 a numerical self-consisted periodic homogenization allows to derive overall elastic properties of long-term degraded samples.

\section{Morphological analysis of sandstone}

Sandstone is a type of sedimentary rock composed of sand-sized grains of minerals. Rock formations that are primarily composed of sandstone usually allow percolation of water or other fluids and are enough porous to store large quantities of them, making them valuable aquifers or petroleum reservoirs. The investigated microstructure of Fontainebleau sandstone, obtained by microtomography, is presented in Figure 1. The initial size of the sandstone geometry is $256 \times 256 \times 256$ voxels with resolution $5.01 \mu \mathrm{m}$ per voxel. In order to characterize morphology of considered microstructure we use

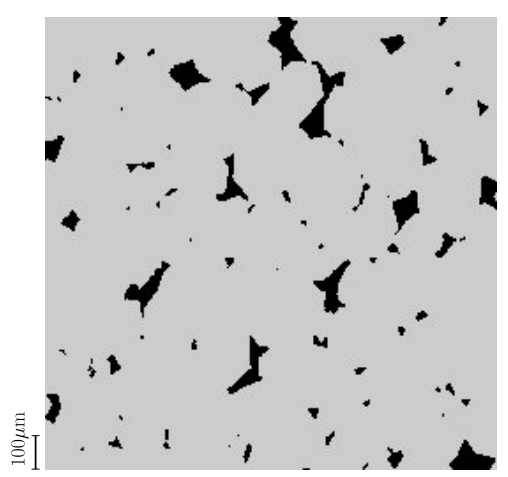

(a) slice of sandstone $\left(256 \times 256 \mathrm{px}^{2}\right)$

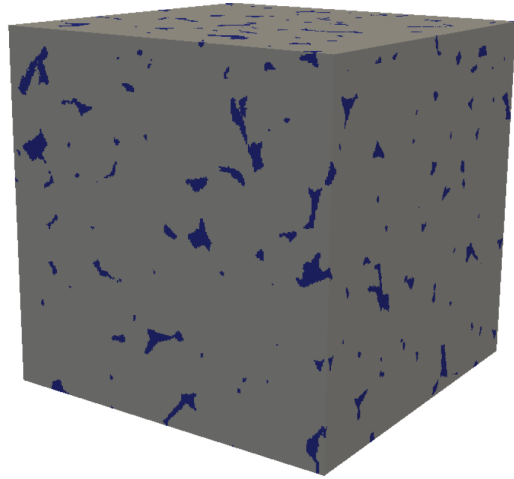

(b) $3 \mathrm{D}$ microstructure $\left(256 \times 256 \times 256 \mathrm{px}^{3}\right)$

Figure 1: Visualisation of a binarized sandstone microstructure as recived. Grey colour corresponds to solid phase, others to porous phase.

mathematical morphology, which provides clear and essential description of images by studying them in the framework of set theory [18, 19]. Using appropriate structuring elements, the image data is converted into some shape characteristics. Let $X$ be an arbitrary set (of $\mathbb{R}^{3}$, e.g.) representing a binary image and $B$ is a subset representing a structuring element. The dilation of $X$ by $B$ is:

$$
X \oplus B=\{x+b \mid x \in X, b \in B\} .
$$

Contrary, the erosion of $X$ by $B$ may be defined as follows:

$$
X \ominus B=\{x \in X \mid x+b \in X, b \in B\} .
$$

Combining erosion and dilation, a morphological opening, is obtained:

$$
X \circ B=(X \ominus B) \oplus B .
$$

This opening acts as a filter and is a basic tool in granulometry analysis. Objects of interest can be highlighted 
using accurate structuring element $B$. Since we have interest for a numerical reconstruction method of real rock sample obtained via microtomography, the following main morphological parameters turn now to be investigated [20]: basic measures (porosity, etc.), sizing, spatial distribution and connectivity.

\subsection{Basic measures}

Basic characteristics of microstructure e.g. porosity, specific surface, number of pores etc. are quantified by global measures. Here we focus on pore volume fraction (porosity). The porosity of the investigated microstructure of Fontainebleau sandstone is equal to $\varphi \simeq 0.0463$. Detailed investigations of others global descriptors can be found in [21].

\subsection{Sizing - granulometry}

The granulomentry of a given phase can be estimated by mathematical morphology $[19,22,23,24]$. The principle of the method consists in performing successive morphological openings with increasing size of the structuring element. After each operation, the change of solid volume fraction $G(r)$ is calculated. To a given structuring element $B$ corresponds a family $B_{r}, r=$ $0,1, \ldots$ of dilated structuring elements $B_{r}=B \oplus S_{r}$, where $S_{r}$ is the sphere of radius $r$. The granulometric function $G_{r}(X)$ of a set $X$ is thus given by:

$$
G_{r}(X)=1-\frac{\left|X \circ B_{r}\right|}{|X|},
$$

where symbol $|\cdot|$ denotes the measure (cardinality) of a set. The volumetric size distribution is defined in following way: $g_{r}(X)=G_{r+1}(X)-G_{r}(X)$ [Fig 2]. It is worth noting that the shape of the initial structuring element $B$ has a direct influence on the results. In order to estimate the granulometry of solid matrix of the Fontainebleau sandstone two different SE are used, cross-shaped (+) and binarised spheres $(\bullet)$ [Fig. 2]. As we can see, both distributions are translated with respect to each other, and their values should be treated only as a rough approximation of real granulometry. However, both mean values, $r_{\text {mean }}^{+}=23.7 \pm 4.9$ and $r_{\text {mean }}^{\circ}=17.4 \pm 3.5$, give a hint for the radius that should be used in the generative process, that we have in mind for the sequel: $r_{\text {mean }} \simeq 20$ px.

\subsection{Spatial distribution - covariance function}

Covariance function has been firstly introduced by G. Matheron $[18,22]$ as tool that provides clear and essential description of spatial distribution. More details

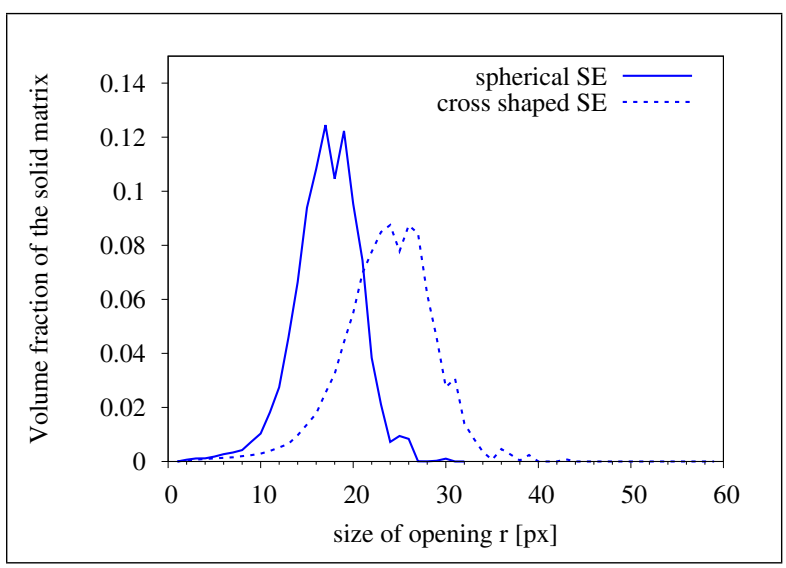

Figure 2: Granulometry of sandstone obtained by two different structuring elements.

and other applications may be found in following references $[19,20,25,26,27]$. We can assume that a binary image representing heterogeneous medium is one of the possible realisations generated by a specific stochastic process $[25,26]$. Among statistical descriptors, the covariogram is a convenient tool to investigate stationary and ergodic media ${ }^{1}$.

For a given set (phase) $X$ and a vector $\vec{h}$, the covariance $C(X, \vec{h})$ is the probability that both an arbitrary point of $X$ and its translation by $\vec{h}$ belong to $X$ :

$$
C(X, \vec{h})=P\{x \in X, x+\vec{h} \in X\} .
$$

Moreover, covariance possesses following properties: $C(X, h=0)=|X|=\varphi$ and $C(X, h \rightarrow+\infty)=|X|^{2}=\varphi^{2}$, where $h=\|\vec{h}\|$ and if $X$ is the porous phase. Since $C(X, \vec{h})$ depends on the direction of the translation vector $\vec{h}$, the covariance is a convenient tool to investigate the anisotropy of a given microstructure.

Figure 3 presents the covariance of the microstructure of Fontainebleau sandstone. Since this covariance is the same in three directions $x, y$ and $z$, we assume in the sequel an overall isotropy of the microstructure of the Fontainebleau sandstone. Moreover, the characteristic size at which covariance function intersects its asymptotical value $\varphi^{2}$ is about $\lambda \simeq 20 \mathrm{px}$. Since covariance function is integrable, the integral range reads for a set

\footnotetext{
${ }^{1}$ ergodic systems possess the same behaviour averaged over time as averaged over the space for each state of the system
} 


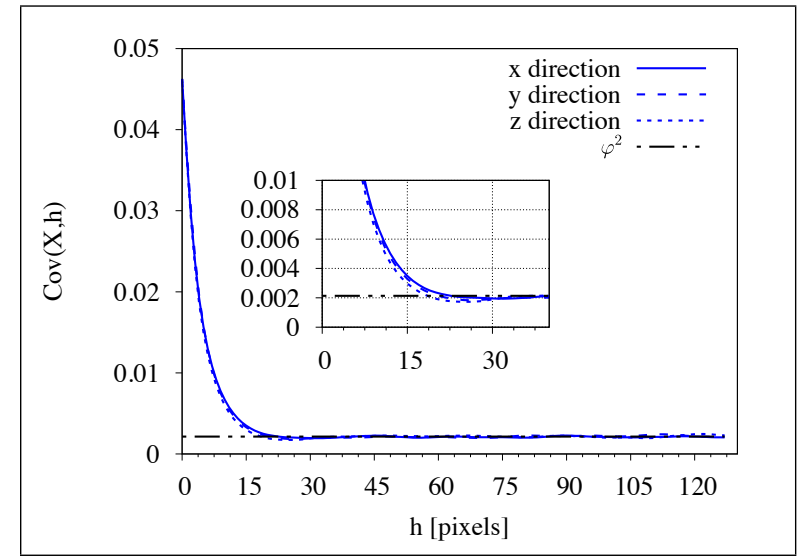

Figure 3: Covariance function of porous phase of investigated sandstone microstructure $(\varphi=4.63 \%)$ plotted for three chosen directions.

$X$ as well as for its complementary set $X^{\mathrm{c}}[26]$ :

$$
\begin{aligned}
& A(X)=A\left(X^{\mathrm{c}}\right)=\frac{1}{C(X, 0)-C(X, 0)^{2}} \times \\
& \times \int_{\mathbb{R}^{3}}\left[C(X, h)-C(X, 0)^{2}\right] \mathrm{d} h .
\end{aligned}
$$

For the Fontainebleau sandstone we obtain:

$$
\begin{aligned}
& A=A(\text { pores })=A(\text { matrix })= \\
& =\frac{1}{\varphi-\varphi^{2}} \int_{\mathbb{R}^{3}}\left[C(X, h)-\varphi^{2}\right] \mathrm{d} h \simeq 1150 \mathrm{px}^{3} .
\end{aligned}
$$

The characteristic scale of each phase is thus: $l=\sqrt[3]{A} \simeq$ $10 \mathrm{px}$.

\subsection{Connectivity - brief analysis}

Mainly there are two different kinds of porosities [28, 29], the first one is a so-called interconnected or effective porosity $\varphi_{\text {eff }}$, which forms percolated pore network and the second one is the isolated or non-interconnected porosity. To extract the percolated porous network Connected Components Labelling (CCL) algorithm was implemented $[30,31]$. The central role is played by definition of pixels connectivity, which can be represented by an arbitrary structuring element. For the purpose of this study, the cross-shaped element has been chosen to define connectivity of $3 \mathrm{D}$ structures. The percolated (effective) porous phase is represented by assembly of pores whose inlet and outlet are localized in opposite faces of geometry. Figure 4 shows the result of the algorithm, which was applied to the microtomography of Fontainebleau sandstone. Analysis of porous phase shows that the microstructure is percolated in all directions and that the effective porosity is $\varphi_{\text {eff }}=0.029$.

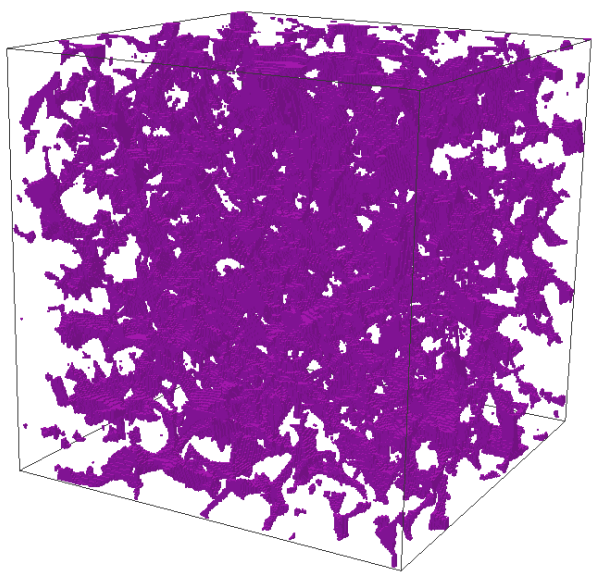

(a) total porous phase; $\varphi=0.046$

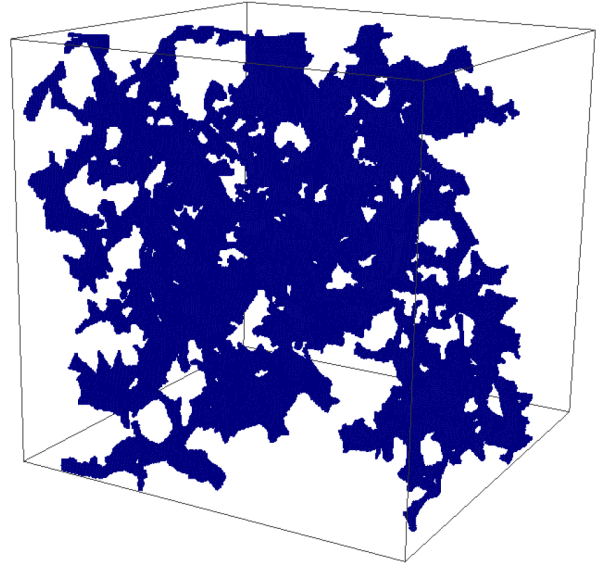

(b) percolated; $\varphi_{\text {eff }}=0.029$

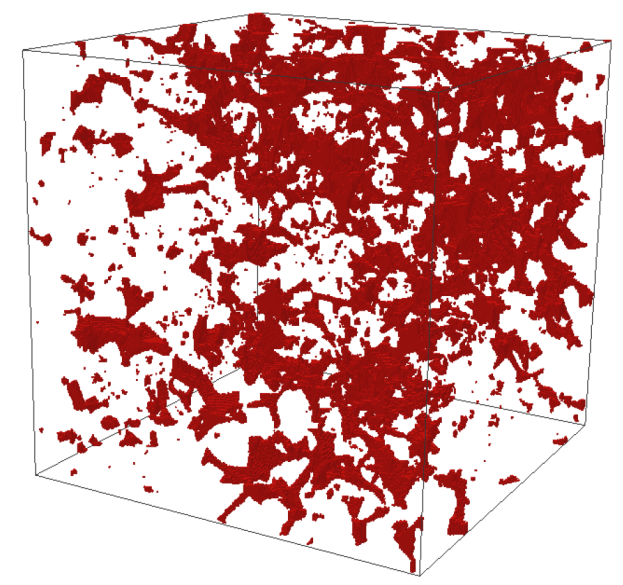

(c) isolated; $\varphi_{\text {iso }}=\varphi-\varphi_{\text {eff }}=0.017$

Figure 4: Repartition of porous phase of the Fontainebleau sandstone. 


\subsection{Conclusions concerning morphological analysis}

The main morphological characteristics of microstructure of sandstone may be presented as follows: overall porosity $\varphi \simeq 4.6 \%$, mean grain radius $r_{\text {mean }} \simeq 20$ px [Fig. 2], spatial isotropy and characteristic size $\lambda \simeq 20$ px [Fig. 3] and percolation in all directions, effective volume fraction (effective porosity) $\varphi_{\text {eff }} \simeq$ $2.9 \%$. Therefore, we propose a method to reconstruct microstructure of sandstone which preserves simultaneously characteristics mentioned above.

\section{Reconstruction method and validation}

The aim is to reconstruct the sample, in a simple and efficient way, by implementing some basic stages inspired by natural formation process of sandstone. Following Bake and Øren [32, 33], the sample reconstruction is obtained by three processes. Grain deposit grain assembly, this stage can be seen as sandgrains transport and sediment. Compaction - bulk volume reduction, this step mimics natural compression. Diagenesis - radii increment, this stage imitates cementation and mineralization process. Each step of the methodology is described below and calibrated in order to respect aforementioned morphological properties.

\subsection{Grain deposit}

Regarding the granulometry of sandstone, which gives only a rough approximation of real granulometry $\left(r \simeq 20 \mathrm{px}\right.$ ), we use three different initial radii $r_{\text {initial }}$ raging from the characteristic scale $l=10 \mathrm{px}$, to the characteristic size $\lambda \simeq 20 \mathrm{px}: 10 \mathrm{px}, 15 \mathrm{px}$ and $20 \mathrm{px}$. The grain size (diameter) thus ranging from $100 \mu \mathrm{m}$ to 200 $\mu \mathrm{m}$ in accordance with [34]. The use of monodisperse spheres is justified by the fact that in 3D each deposit of spherical grains is fully percolated. The grains are placed one by one into a three-dimensional box according to the algorithm of gravitational potential minimization [35], implemented in LMGC90 [36, 37].

\subsection{Compaction}

Corresponding samples present loose packing of rigid spheres. During compaction, friction coefficient is set to zero, to facilitate rearrangements of the grains. This step is coupled with the next one - diagenesis. Because of its random nature, diagenesis may have a significant influence on the isotropy of the system. Therefore, in order to reduce that effect we decided to apply a compaction step. In the paper we investigate three different scenarii: triaxial compaction, sedimentation (compaction under gravity) and no compaction.

\subsection{Diagenesis}

In general, aim of diagenesis is to control porosity of a specimen by increasing radii of grains. In order to avoid border effects and regular packing of spheres, porosity is measured in a window $\left(256 \times 256 \times 256 \mathrm{px}^{3}\right)$ localized inside, in the center of the 3D box. Even if the initial deposit is composed of monodisperse grains, the diagenesis process induces polydispersity. Therefore, diagenesis process consists of two steps. First step is an uniform radii increment by the value of $1 \mathrm{px}$, until the following criterion is satisfied:

$$
\varphi^{\text {window }} \leq(1+\Delta) \times \varphi^{\text {sandstone }},
$$

where $\varphi^{\text {window }}$ denotes the porosity measured in the window, $\varphi^{\text {sandstone }}$ is the porosity of the reference sample and $\Delta$ is a chosen error. The second step, consist in radius increment by 1 px of randomly chosen grains. This step takes place until porosity is adjusted to the desired one. The corresponding pseudo-code reads:

choose errors $\Delta$ and $\epsilon$, typically $\Delta=0.8$ and $\epsilon=1 \times 10^{-4}$ while $\varphi^{\text {window }}>(1+\Delta) \times \varphi^{\text {sandstone }}$

[increment by $1 \mathrm{px}$ the radius of all grains] while $\varphi^{\text {window }}>(1 \pm \epsilon) \times \varphi^{\text {sandstone }}$

[increment by $1 \mathrm{px}$ the radius of randomly chosen grain (draw with replacement)]

\subsection{Choosing the initial radius and compaction sce- nario}

In order to chose the appropriate initial radius of the grain deposit and scenario of compaction, we analyse covariance functions presented in Figure 5. Each figure presents covariances plotted for all chosen different initial values of radii for each scenario of compaction. For the sake of clarity only three chosen directions are shown. The letters $\mathrm{C}, \mathrm{S}, \mathrm{G}$ denote triaxial compaction, sedimentation and geometrical (no compaction) deposit respectively. The numbers 10 [blue], 15 [red] and 20 [green], correspond to different initial radii of grain deposit and the black curve is the reference covariance function of Fontainebleau sandstone. Figure 5 (a) presents covariance of triaxial compaction scenario. We may see that the covariance of Fontainebleau sandstone lays between the covariances representing deposits of initial radii of $10 \mathrm{px}$ and $15 \mathrm{px}$. Results obtained for other scenarii give the same conclusion, therefore for the sake of simplicity we showed only the covariance of triaxial compaction. Regarding Figure 5 (b) and approaching to initial radii of $10 \mathrm{px}$ we can see that the most isotropic geometry is obtained by applying triaxial compaction. 


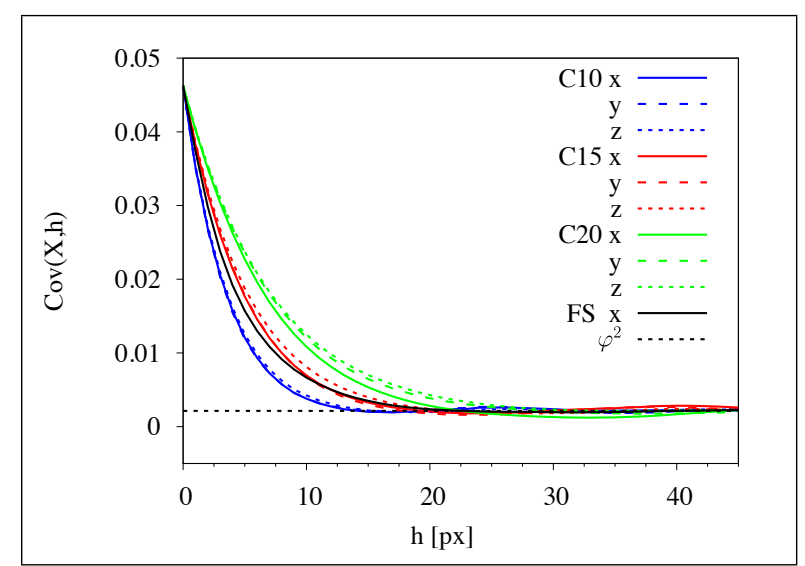

(a) choosing initial radius - covariances functions plotted for all initial values of radii of triaxial compaction

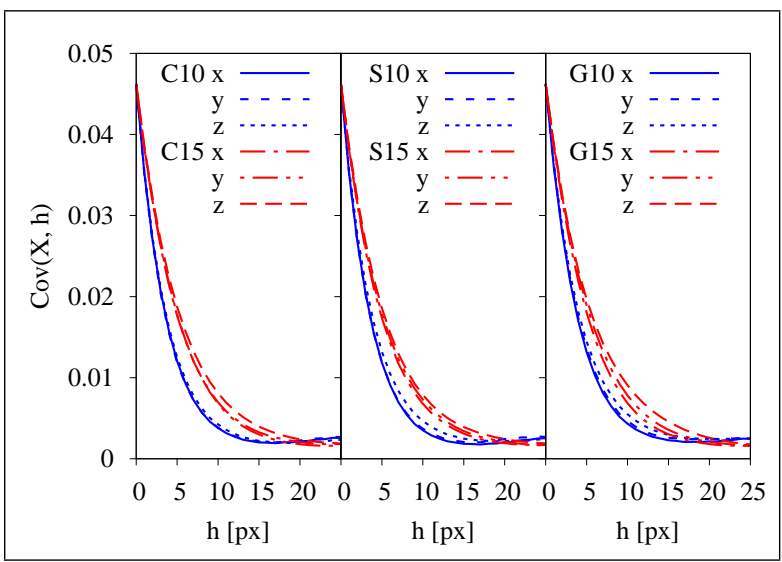

(b) choosing compaction scenario - covariances function plotted for initial values of radii: 10 and $15 \mathrm{px}$, for each scenario of compaction

Figure 5: Covariance function of deposits as a descriptor used to chose initial radius and compaction scenario.

\subsection{Analysis of anisotropy of contacts}

To dispel any doubts concerning the choice of the scenario of compaction, we present inhere a brief analysis of anisotropy of contacts. Within the framework of contact dynamic, it is convenient to use fabric tensor $F$ [38] to characterize the distribution of normal directions $n^{c}$ at contacts. Fabric tensor is given by:

$$
F=\frac{1}{N_{c}} \sum_{c=1}^{N_{c}} n^{c} \otimes n^{c},
$$

where $n^{c}$ is unit vector representing the normal direction of the force at contact, $\otimes$ is the dyadic product and $N_{c}$ is the number of contacts. The anisotropy parameter $a_{c}$ of fabric tensor is characterized by:

$$
a_{c}(F)=1-\frac{\|F: I-F\|}{\|F\|},
$$

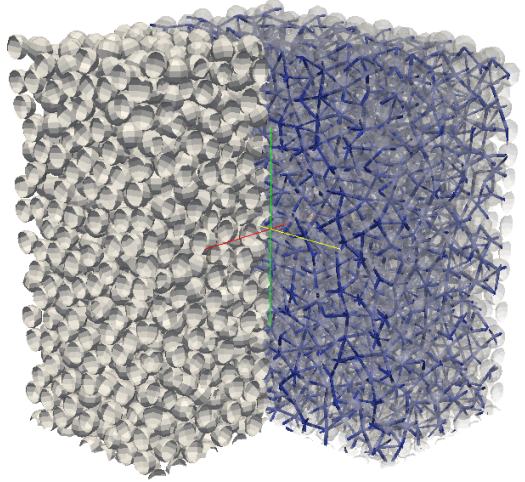

(a) sedimentation scenario and normal forces distribution; $r_{\text {initial }}=15 \mathrm{px}$

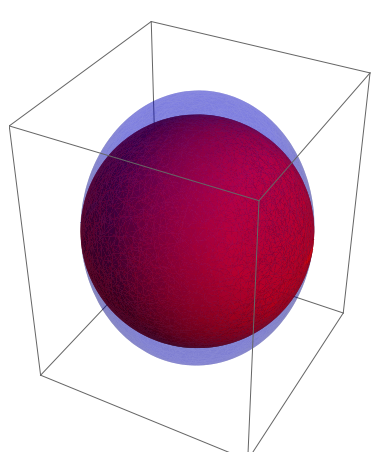

(b) transformation of the unit sphere (red) by the fabric tensor (blue)

Figure 6: Visualisation of normal forces distribution of sedimentation scenario and of associated fabric tensor; the anisotropy parameter $a_{c}=0.982$.

where $I_{i j}=\delta_{i j}$, with $\delta$ the Kronecker delta and $\|F\|=$ $\sqrt{F: F}=\sqrt{F_{i j} F_{i j}}$ for any second order tensor. We investigate distributions of contacts for sedimentation scenario S15 [Fig. 6] and triaxial compaction C15 [Fig. 7] in both cases $r_{\text {initial }}=15 \mathrm{px}$. The anisotropy parameter $a_{c}(F)$ are equal 0.982 and 0.999 for $\mathrm{S} 15$ and $\mathrm{C} 15$ respectively. The analysis of contacts anisotropy confirms that the scenario of triaxial compaction with initial radius $r_{\text {initial }}=15 \mathrm{px}$ gives the most isotropic results. Moreover, refining the study around $15 \mathrm{px}$, we found that the most accurate results is obtained for $r_{\text {initial }}=14 \mathrm{px}$.

\subsection{Validation of generated samples}

Finally, to generate the equivalent microstructures, we have chosen $r_{\text {initial }}=14 \mathrm{px}$ and triaxial compaction scenario. Each realisation is obtained from the same, previously compacted, grain deposit. Nevertheless, the diagenesis process, consisting of random radius incre- 


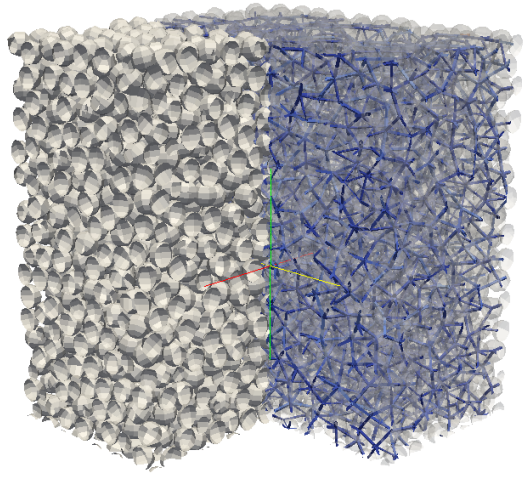

(a) triaxial compaction scenario and normal forces distribution; $r_{\text {initial }}=15 \mathrm{px}$

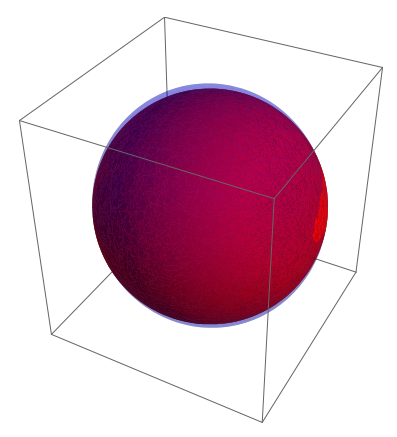

(b) transformation of the unit sphere (red) by the fabric tensor (blue)

Figure 7: Visualisation of normal forces distribution of triaxial compaction and of associated fabric tensor; the anisotropy parameter $a_{c}=0.999$.

ment, allows us to obtain morphologically different microstructures. The generated samples are subjected to rough sorting process, which consists of two filters involving percolation and effective porosity. The first filter is percolation - samples which are not percolated in all directions are excluded from the further analysis. And the second one, effective porosity - samples which are not satisfying the following condition are excluded: $\varphi_{\text {eff }}=(1 \pm \tilde{\epsilon}) \times \varphi_{\text {eff }}^{\text {sandstone }}$, where $\varphi_{\text {eff }}^{\text {sandstone }}$ is the effective porosity of the reference sample of Fontainebleau sandstone and $\tilde{\epsilon}=5 \times 10^{-2}$. According to the aforementioned conditions, 10 accurate samples are generated with about $18 \mathrm{~min}$ CPU time per realisation [2.6 $\mathrm{GHz}$ Intel Core i7, non-parallel code].

In Figures 8 we can see the microstructure of one generated sample. Regarding also Figure 1 (a) and Figure 4 (a), we can see that the porous network of reconstructed sample is a good approximation of the real one. Figure

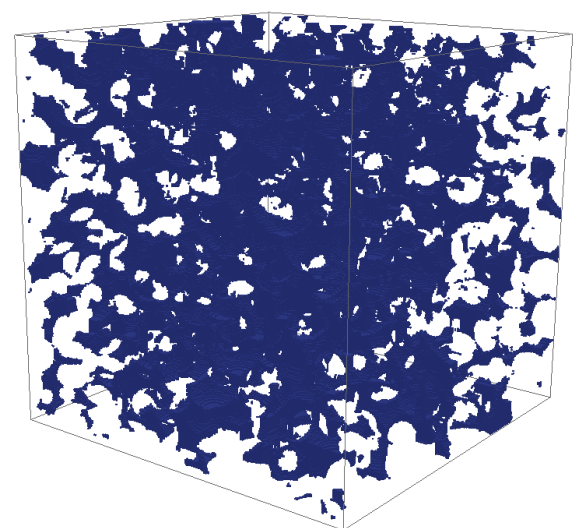

(a) 3D pore network of selected generated sample

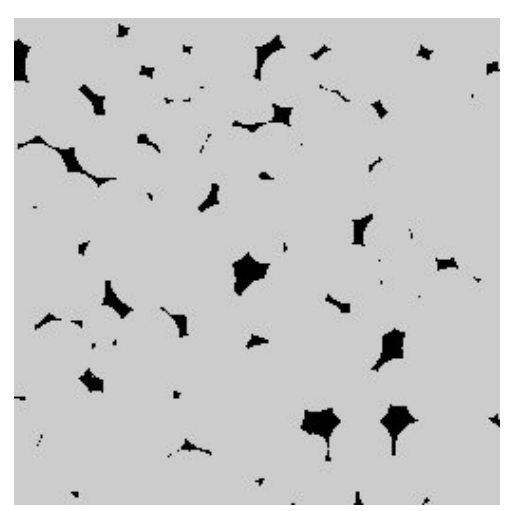

(b) 2D image, plane XY

Figure 8: Visualisation of one of generated samples.

9 presents the covariance function, both of the sandstone (FS) and the averaged covariance over 10 generated samples (GS). For the sake of clarity, we present only result obtained for $\vec{z}$ direction. We can notice a very good spatial correlation between generated specimens and the real microstructure. The maximal standard deviation of the covariance function for the 10 samples is about $s \simeq 2 \times 10^{-4}$. This argument ensures the accuracy of the numerical estimates based on the generated samples (see eg. Kanit et al. [39])

Figure 10 presents comparison of grain size distribution of sandstone and averaged values obtained for generated samples. Although, the methodology of reconstruction is not strictly based on fitting the granulometry of sandstone, the results remains in good concordance. In order to summarise the data in a more compacted way, Table 1 presents the considered morphological de- 


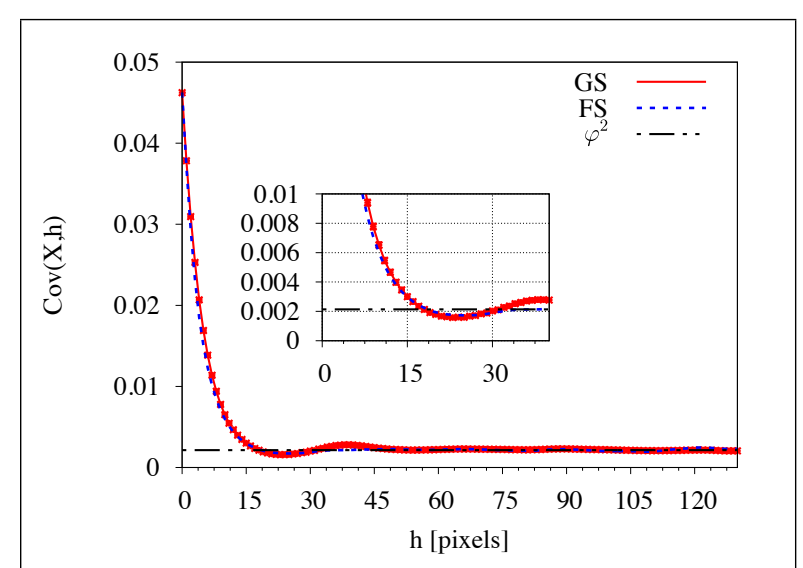

Figure 9: Comparison of covariance function of sandstone (FS) and averaged covariance of generated samples (GS) plotted for direction $\vec{z}$.

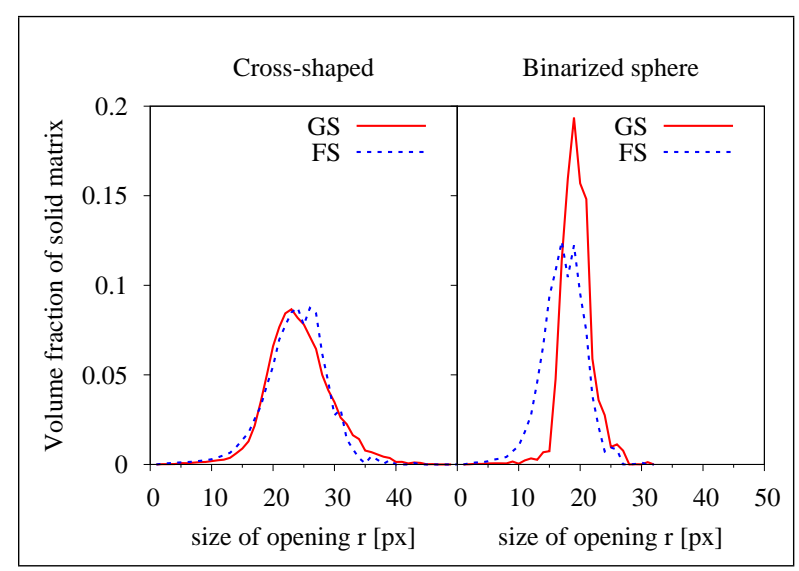

Figure 10: Comparison of granulometry of sandstone (FS) and averaged granulometry obtained for generated samples (GS) using crossshaped and spherical structuring elements.

scriptors of Fontainebleau sandstone and averaged values over the 10 generated samples. A good agreement

Table 1: Summary of morphological validation of generated microstructures vs. sandstone. Where $\langle\mathrm{GS}\rangle$ denotes the mean over 10 samples; $\varphi_{\text {eff }}$ stands for effective porosity; $S_{t}, S_{t}^{e f f}$ is specific surface and effective specific surface; $\lambda$ denotes characteristic length; $r_{\text {mean }}^{\bullet}$ and $r_{\text {mean }}^{+}$describe mean radii obtained by binarized spheres and cross-shaped structuring element respectively.

\begin{tabular}{l|l|l|l}
\hline Descriptor & FS & $\langle\mathrm{GS}\rangle$ & St. dev. \\
\hline$\varphi_{\text {eff }}$ & 0.029 & 0.028 & 0.001 \\
\hline$S_{t}[1 / p x]$ & 0.051 & 0.050 & 0.001 \\
\hline$S_{t}^{e f f}[1 / p x]$ & 0.029 & 0.028 & 0.001 \\
\hline$\lambda$ & 23 & 21 & 1 \\
\hline$r_{\text {mean }}^{\bullet}$ & 17.4 & 19.4 & 0.3 \\
\hline$r_{\text {mean }}^{+}$ & 23.7 & 24.3 & 0.4
\end{tabular}

is also noticed for these mean values. Since the standard deviations are less than 5\%, we consider in the sequel that each of the 10 generated samples can be seen as one statistically equivalent sample of Fontainebleau sandstone.

\section{Numerical dissolution}

Binarized geometry of natural rock gives only approximation of real connectivity. Therefore, we investigate two different scenarii of dissolution. The proposed numerical model of dissolution is based on morphological dilation of the porous phase using cubic structuring element. The choice of a 3D isotropic structuring element [23] can be justified by far field and long term hypothesis, when concentration of reactants is assumed to be homogeneously distributed in the pores space at the sample scale $[6,8]$. Hence, at each iteration (dissolution step) one operation of dilation if performed. Two different scenarii are considered.

Isotropic dissolution - first scenario, which consists in dissolving all void spaces even if pores are isolated. If the set $X_{0}$ represents binary image of the initial porous phase, the results of $i$-th step of isotropic dissolution is recursively defined using a structuring element $B$ :

$$
X_{i}=X_{i-1} \oplus B
$$

Dissolution of percolated network - second scenario, which consists in dissolving only the percolated porous network. Denoting by $\Xi(\cdot)$ an operator acting on a binary image and returning its percolated porous network, the results of the $i$-th step of percolated network dissolution is defined as:

$$
X_{i}=\left[\Xi\left(X_{i-1}\right) \oplus B\right]+X_{0} .
$$

This modelling can be adjusted to any time-dependent process, as e.g. precipitation of minerals.

Dissolution processes, described by eq. (9) and (10) are performed in 3D and are stopped when porosity reach about $40 \%$, which corresponds to about 4 and 6 iterations for the case of isotropic and percolated porous network dissolution respectively. In order to analyse influence of numerical dissolution on the samples morphology, Figure 11 presents the evolution of the covariance function for $\vec{x}$ direction.

Analysing characteristic size $\lambda$, two important conclusions are noticed. First, during isotropic dissolution of the entire network, characteristic size of microstructure remains constant, while for the second scenario the size increases significantly. Second, for both cases we 


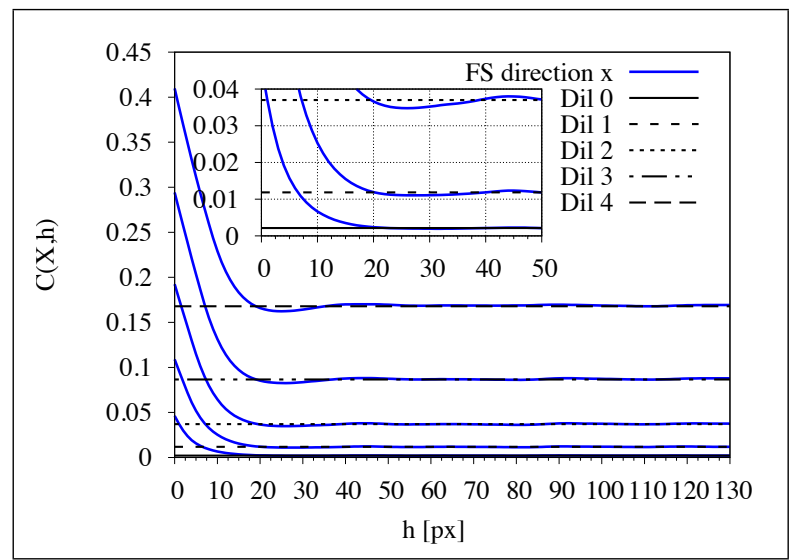

(a) Fontainebleau sandstone - isotropic dissolution

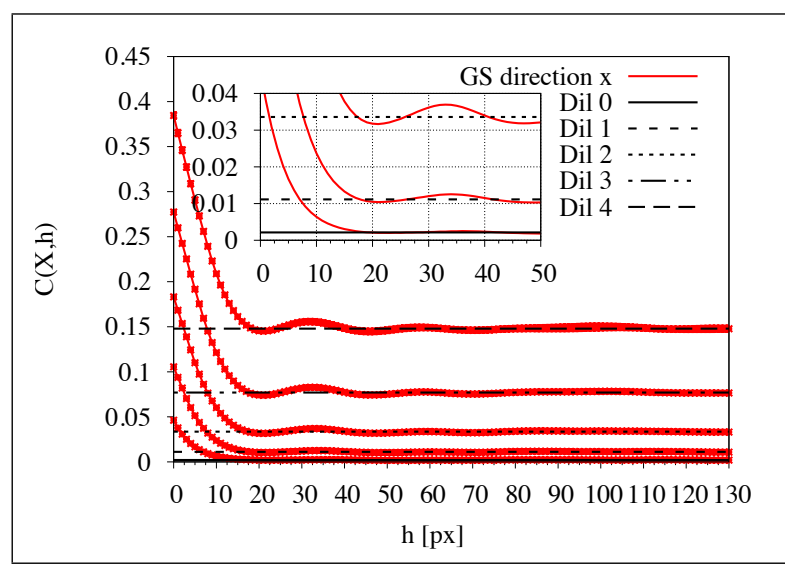

(c) Numerical samples - isotropic dissolution

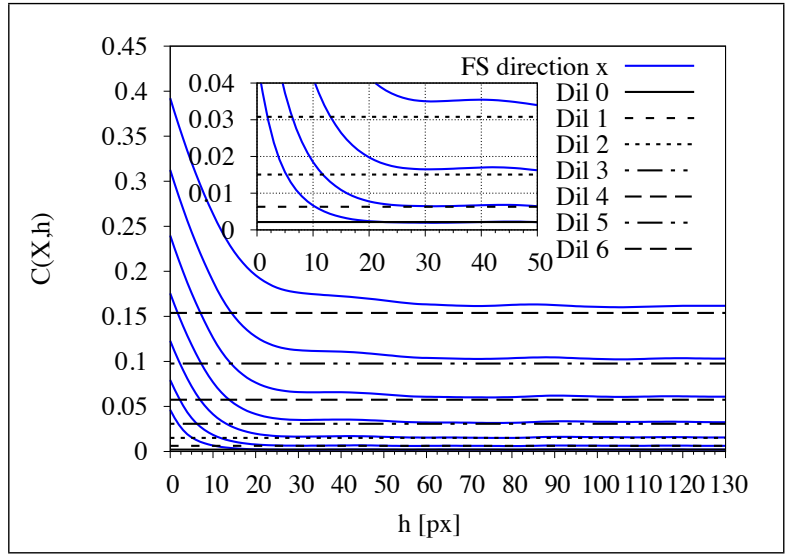

(b) Fontainebleau sandstone - network dissolution

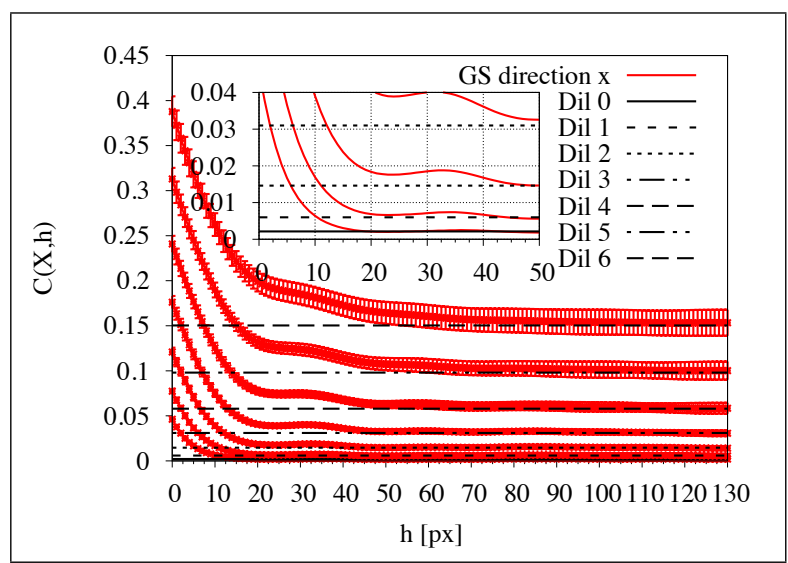

(d) Numerical samples - network dissolution

Figure 11: Evolution of covariance function for Fontainebleau sandstone and the generated samples for two scenarii of dissolution. Dil $i$ corresponds to theoretical asymptote referring to the $i$-th step of dissolution.

can assume that size of considered samples is sufficient to consider them as RVEs. It is also important to remark, that evolutions of covariances for both materials (real and generated), for each scenario of dissolution, possess very similar character but the standard deviation of the covariance function for the 10 generated samples is larger in the case of network dissolution than in the case of isotropic dissolution. It could be easily showed, by analysing other morphological descriptors, that the morphological equivalency of generated samples in regard of real one remains true even during the dissolution processes.

\section{Numerical estimation of overall elastic moduli}

One of the tools that allows us to obtain effective properties is the so-called periodic homogenization $[40,41]$. This classical approach treats heteroge- neous media as materials possessing hierarchical structure which can be described by a finite number of separable scales. For the purpose of this study, two levels are used: micro and macro-scale. Effective properties (macro-scale) are assumed to be homogeneous and can be estimated through averaging process. This approach takes into account all interactions between phases forming heterogeneous structure at micro-scale. Aforementioned method is implemented using a finite element method - Cast $3 \mathrm{M}^{2}$.

\subsection{Periodization - fixed point method}

In this work we consider periodic boundary conditions prescribed on RVE cell within strain approach framework [40, 41]. However, since we are dealing with

\footnotetext{
${ }^{2}$ http://www-cast3m.cea.fr
} 
images of rocks or generated samples, geometrical periodicity is not achieved in a direct way. To solve this problem we add a layer around the extracted cubic cell and we adopt a fixed point method. Initially, the stiffness tensor of the layer is assumed to be equal to the stiffness tensor of the solid phase. During the fixed point method the fourth order stiffness tensor of the layer $C^{\text {layer }}$ is updated:

$$
C^{\text {layer }}[i]=C^{\text {hom }}[i-1],
$$

where $i$ denotes iteration number and $C^{\text {hom }}$ is the overall fourth order stiffness tensor of the RVE. The stopping criterion $\gamma^{\text {stop }}$ for this method is defined by:

$$
\gamma^{\text {stop }}=\frac{\left\|C^{\text {layer }}[i]-C^{\text {hom }}[i]\right\|}{\left\|C^{\text {hom }}[i]\right\|}<10^{-4},
$$

where $\|D\|=\sqrt{D:: D}=\sqrt{D_{i j k l} D_{i j k l}}$ for any fourth order tensor. The choice of $\gamma^{\text {stop }}$ value is justified in Figure 12, since overall elastic properties are constant for $-\log \left(\gamma^{\text {stop }}\right)>4$.

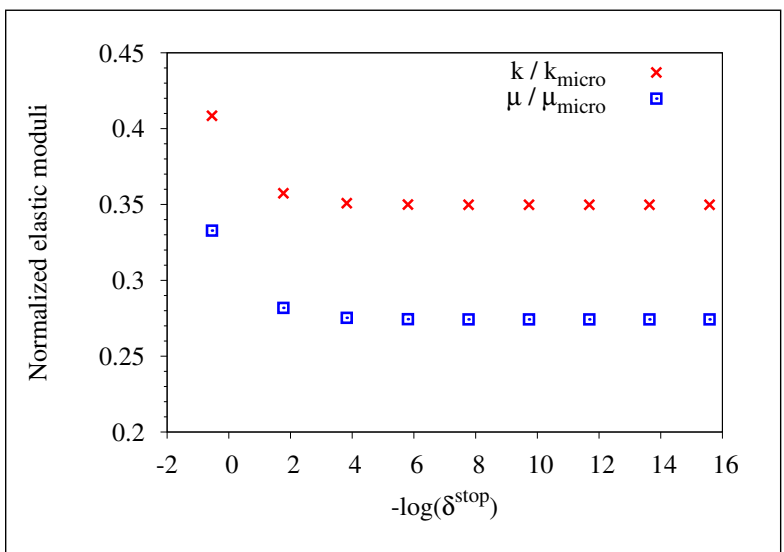

Figure 12: Overall normalised elastic moduli (bulk $k^{\text {hom }}$ and shear $\mu^{\text {hom }}$ ) as a function of $\gamma^{\text {stop }}$. Results obtained for geometry representing 6-th degraded state of Fontainebleau sandstone in case of percolated network dissolution.

When desired convergence is achieved, effective stiffness tensors are projected on the classical isotropy basis in order to extract bulk $k$ and shear $\mu$ moduli: $3 k^{\text {hom }}=C^{\text {hom }}:: J$ and $2 \mu^{\text {hom }}=\frac{1}{5} C^{\text {hom }}:: K$, where $J_{i j k l}=\frac{1}{3}\left(\delta_{i j} \delta_{k l}\right), I_{i j k l}=\frac{1}{2}\left(\delta_{i k} \delta_{j l}+\delta_{i l} \delta_{j k}\right)$ and $K=I-J$.

\subsection{Discretization and its influence}

A microstructure defined by a binary image is already discretized. A regular cubic mesh can thus be constructed based on appropriated voxel: the larger the number of voxels, the finer the mesh. Let $M$ denotes the number of cubic mesh elements along the edge of the cubic RVE. It is shown that discretisation error for FE methods of any elastic moduli can be estimated according to the following formula $[42,43,44,45]$ :

$$
P(M)=P_{0}+\frac{a}{M},
$$

where $P(M)$ is the computed elastic property and $P_{0}$ is so-called continuum value, which corresponds to the case when $M \rightarrow \infty$. To estimate $P_{0}, P(M)$ is computed at least for three different values of $\mathrm{M}$ and then a linear function is fitted according to the least squares method [Fig. 13].

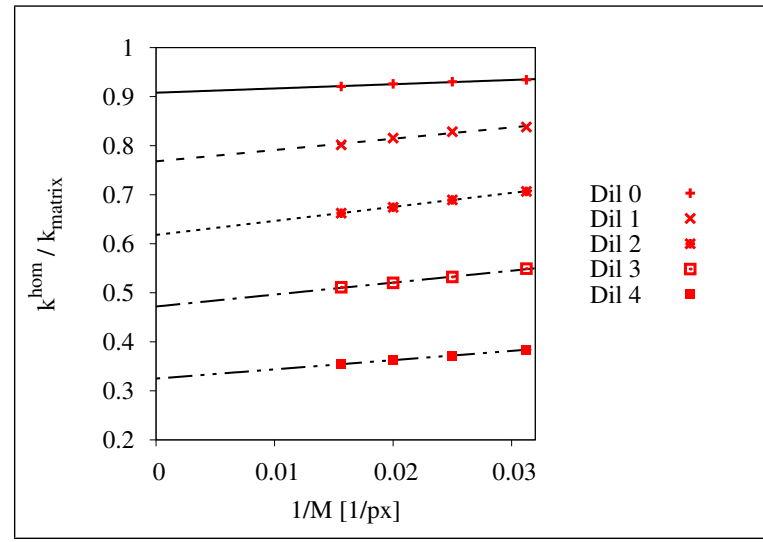

Figure 13: Computed normalized bulk modulus $k^{\text {hom }} / k_{\text {matrix }}$ as a function of resolution $1 / M$. Results obtained for Fontainebleau sandstone for isotropic scenario. Dil $i$ corresponds to the $i$-th step of dissolution.

\subsection{Robustness of the results}

The elastic properties of the solid phase are taken form Mavko et al. [46]: bulk modulus is equal $k_{\text {matrix }}=$ $37.0 \mathrm{GPa}$ and the shear modulus $\mu_{\text {matrix }}=44.0 \mathrm{GPa}$. The corresponding Young modulus and Poisson ratio are: $E_{\text {matrix }}=94.53 \mathrm{GPa}, v_{\text {matrix }}=0.0742$. Concerning the aforementioned method, the precision of the results can depend both of: the contrast between the Young moduli of solid matrix and pores $\kappa=E_{\text {matrix }} / E_{\text {pores }}$ and the layer and its thickness $T$. In [21] it has been shown that this method converges and that influence of layer thickness on overall elastic properties is negligible.

The influence of $\kappa$ arises from the potentially illconditioned finite element stiffness matrix. Since the property of a cubic mesh corresponds to the phase associated to the voxel, the boundaries of the porous phase are not explicitly meshed. A small value of Young modulus $E_{\text {pores }}$ is affected to the meshes associated to the porous phase, small compared to the value of the Young modulus of the solid phase $E_{\text {matrix }}$. The contrast $\kappa$ has thus to tend to infinity and in practice to a huge value. 
However, when $\kappa$ increases, the porous phase is better represented, but the finite element stiffness matrix is worse conditioned. Since the Poisson ratios of phases are set to be equal, the influence of $\kappa$ is studied.

We perform computations for last degraded states of sandstone geometry of each dissolution scenario. $\mathrm{Nu}-$ merical results are obtained for entire sample of size $256 \times 256 \times 256 \mathrm{px}^{3}$ scaled by factor four. What means that size of the geometry is $64 \times 64 \times 64 \mathrm{px}^{3}$. In this study we investigate 10 different contrast values ranging from $10^{1}$ to $10^{10}$. Figure 14 presents bulk and shear moduli normalised by their values obtained for maximal considered contrast $\kappa=10^{10}$. We can see that convergence is obtained for $\kappa \approx 10^{4}$.

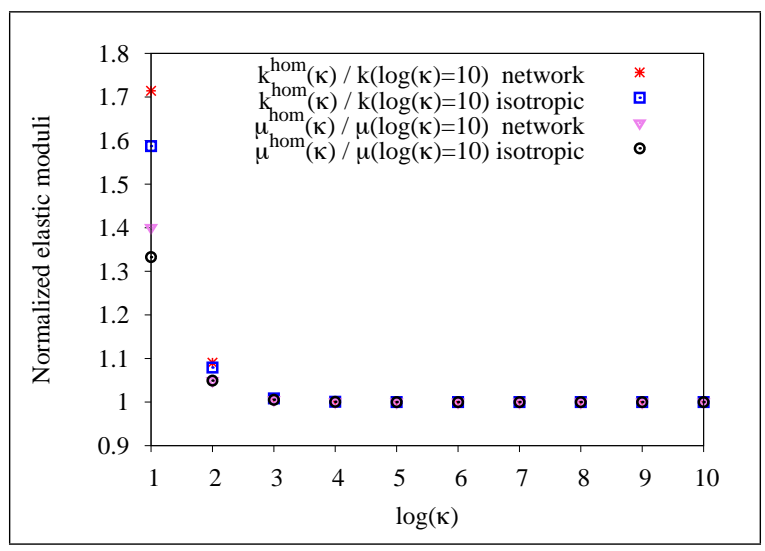

Figure 14: Normalised elastic moduli as a function of contrast $\kappa$ (network - percolated network dissolution; isotropic - isotropic dissolution). The normalizing factors are values of bulk $k$ and shear $\mu$ moduli obtained for the last degraded state of sandstone and for the contrast $\log (\kappa)=10$.

\subsection{Results}

Aforementioned methodology is applied for each considered sample at different dissolved states. For this purpose we investigate entire geometry of sandstone and degraded samples. Following resolutions $\mathrm{M}$ (defined in Section 5.2): $M=64 \mathrm{px}, M=50 \mathrm{px}, M=40$ px and $M=32$ px are used. Extracted continuum values of elastic moduli $(M \rightarrow \infty)$, for both scenarii of dissolution and results obtained by Arns et al. [1], are presented in Figure 15. In their paper [1], different realisations of sandstone microstructure were extracted from four images representing real core samples and the porosity is varying from 0.055 up to 0.24 .

At the first sight, evolution of elastic properties as a function of porosity [Fig.15] for sandstone and the generated samples are in excellent consistency (for each scenario). In general, for both scenarii, shear modulus decreases more significantly than bulk modulus.

Although different mechanism responsible for porosity variation, our results and those presented by Arns et al. [1] show very similar trend In other words, in the work [1] the elastic properties are presented in function of initial porosity in our case it is a function of dissolution induced porosity, so the variation of porosity is driven by evolving microstructure.

In Figure 15, the comparison of (a) and (b), (c) and (d) shows that results obtained for both scenarii of dissolution are very similar. The obtained results as well as those of Arns et al. [1] are in coherence with the selfconsistent estimate [47] which often represents media with total disorder.

\section{Conclusions}

This work presents an efficient and simple method to generated equivalent microstructures of heterogeneous media, that takes into account chosen morphological properties and is inspired by natural process of the formation of sandstone. To estimate equivalent constitutive behaviour, we have also presented a solution using additional layer associated with a fixed point method that enables to impose periodic boundary conditions on non-periodic geometries. A complete methodology to estimate evolution of mechanical behaviour from generated samples morphologically equivalent to microtomographies was presented. Modelling of dissolution phenomenon and the final results shows that a sophisticated modelling of the dissolution process will not affect significantly the results.

\section{Acknowledgements}

We are specially grateful to Dr Philippe Gouze, who provided the CT image of Fontainebleau sandstone microstructure.

[1] C. H. Arns, M. A. Knackstedt, W. V. Pinczewski, E. J. Garboczi, Computation of linear elastic properties from microtomographic images: Methodology and match to theory and experiment., Journal of Geophysics 67 (2002) 1396-1405.

[2] B. Metz, O. Davidson, H. de Coninck, M. Loos, L. Meyer (Eds.), IPCC Special Report on Carbon Dioxide Capture and Storage, Cambridge University Press, Cambridge, United Kingdom and New York, USA, 2005.

[3] L. Luquot, P. Gouze, Experimental determination of porosity and permeability changes induced by massive injection of $\mathrm{CO}_{2}$ into carbonate reservoirs, Chemical Geology 265 (2009) 148159.

[4] P. Gouze, L. Luquot, X-ray microtomography characterization of porosity, permeability and reactive surface changes during dissolution, Journal of Contaminant Hydrology 120-121 (2011) 45-55. 


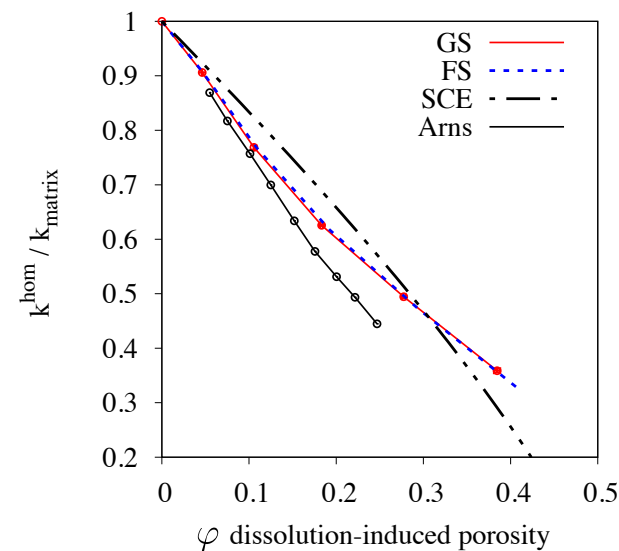

(a) normalized bulk modulus - isotropic dissolution

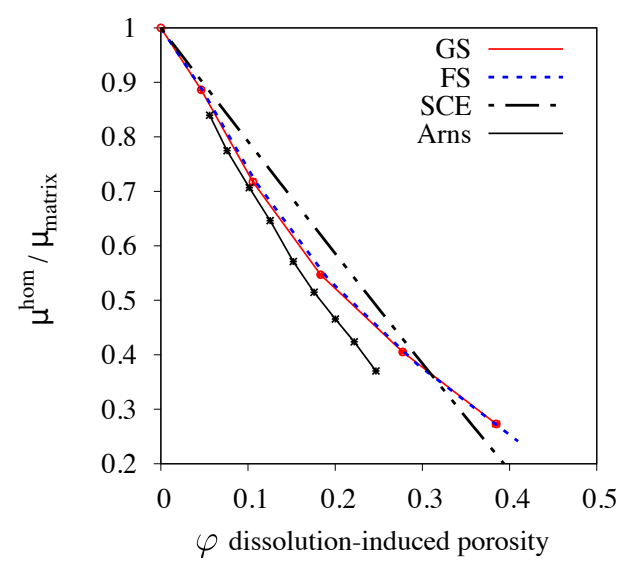

(c) normalized shear modulus - isotropic dissolution

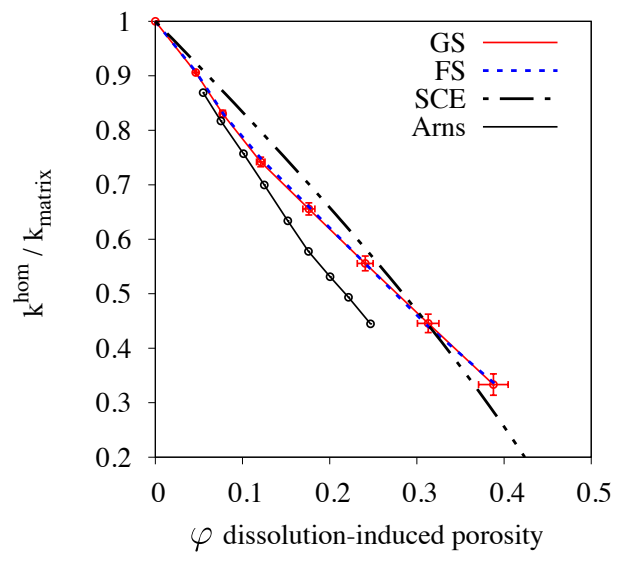

(b) normalized bulk modulus - percolated network dissolution

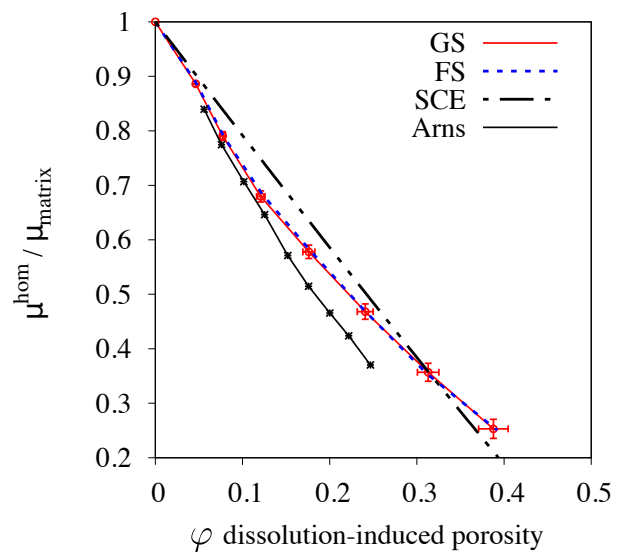

(d) normalized shear modulus - percolated network dissolution

Figure 15: Evolution of normalised elastic moduli of sandstone (FS), averaged over all generated samples (GS) (with standard deviations) for both scenarii of numerical dissolution; comparison with self-consistent estimate (SCE) and with results obtained by Arns et al. [1].

[5] P. Egermann, S. Bekri, O. Vizika, An integrated approach to assess the petrophysical properties of rocks altered by rock/fluid interactions $\left(\mathrm{CO}_{2}\right.$ injection), Paper SCA Presented at the Society of Core Analysts Symposium, Toronto, Canada.

[6] P. Egermann, E. Bemer, B. Ziszner, An experimental investigation of the rock properties evolution associated to different levels of $\mathrm{CO}_{2}$ injection like alternation processes, Paper SCA Presented at the Society of Core Analysts Symposium, Trondheim, Norway.

[7] O. Izgec, B. Demiral, H. Bertin, S. Akin, $\mathrm{CO}_{2}$ injection into saline carbonate aquifer formations I: laboratory investigations, Transport in Porous Media 72 (2008) 1-24.

[8] J. Bemer, J. M. Lombard, From injectivity to integrity studies of $\mathrm{CO}_{2}$ geological storage. chemical alteration effects on carbonates petrophysical and geomechanical properties, Oil \& Gas Science and Technology 65 (2009) 445-459.

[9] M. T. Nguyen, J. Bemer, L. Dormieux, Micromechanical modeling of carbonate geomechanical properties evolution during acid gas injection, Paper ARMA Presented at the 45th U.S. Mechanics/Geomechanics Symposium, San Francisco, USA.
[10] M. A. Peter, Homogenisation in domains with evolving microstructure, Comptes Rendus Mécanique 335 (2007) 357-362.

[11] S. Meier, M. Peter, M. Bhm, A two-scale modelling approach to reactiondiffusion processes in porous materials, Computational Materials Science 39 (1) (2007) 29 - 34, proceedings of the 15 th International Workshop on Computational Mechanics of MaterialsThe 15th International Workshop on Computational Mechanics of Materials.

[12] J. Lewandowska, Modeling by homogenization of the long-term rock dissolution and geomechanical effects, in: G. PijaudierCabot, J.-M. Pereira (Eds.), Geomechanics in $\mathrm{CO}_{2}$ storage facilities, Wiley-ISTE, 2012, Ch. 8, pp. 135-160.

[13] B. Coussy, Poromechanics, John Wiley and Sons Ltd., Chichester, England, 2004.

[14] C. Doughty, K. Preuss, Modeling supercritical carbon dioxide injection in heterogeneous porous media, Vadose Zone Journal 3 (2004) 837-847.

[15] A. Kumar, R. Ozah, M. Noh, G. A. Pope, Reservoir simulations of $\mathrm{CO}_{2}$ geological storage in deep saline aquifers, SPE Journal 10 (2005) 336-348. 
[16] S. D. Hovorka, S. M. Benson, C. Doughty, Measuring permanence of $\mathrm{CO}_{2}$ storage in saline formations: the frio experiment, Environmental Geoscience 13 (2006) 105-121.

[17] K. Wojtacki, J. Lewandowska, P. Gouze, A. Lipkowski, Numerical computations of rock dissolution and geomechanical effects for $\mathrm{CO}_{2}$ geological storage, International Journal for Numerical and Analytical Methods in Geomechanics 39 (2015) 482-506.

[18] G. Matheron, Random Set and Integral Geometry, Wiley, New York, USA, 1975

[19] J. Serra, Image Analysis and Mathematical Morphology Vol. 1, Academic Press, New York, USA, 1982.

[20] D. Jeulin, Random texture models for material structures, Statistics and Computing 10 (2000) 121-132.

[21] K. Wojtacki, Coupling between transport, mechanical properties and degradation by dissolution of rock reservoir, PhD Thesis, University of Montpellier, France, 2015.

[22] S. Matheron, Eléments pour une théorie des milieux poreux, Masson, Paris, 1967.

[23] P. Soille, Morphological Image Analysis Principles and Applications, Springer-Verlag, Berlin, Heidelberg, New York, 2004.

[24] L. Najman, H. Talbot (Eds.), Mathematical Morphology From Theory to Applications, ISTE Ltd. and John Wiley \& Sons Inc., United Kingdom, USA, 2010.

[25] S. Torquato, Random Heterogeneous Materials, Springer, New York, USA, 1982

[26] C. Lantuéjoule, Geostatistical Simulations Models and Algorithms, Springer, Berlin, Gemany, 2002.

[27] A. Nait-Ali, O. Kane-Diallo, S. Castagnet, Catching the time evolution of microstructure morphology from dynamic covariograms., Comptes Rendus Mécanique 343 (2015) 301-306.

[28] F. Dullien, Porous Media Fluid Transport and Pore Structure, Academic Press, San Diego, USA, 1991.

[29] J. Bear, Dynamics of fluids in porous media, Dover Publications, New York, USA, 1972

[30] S. Marchand-Maillet, Y. M. Sharaiha, Binary Digital Image Processing, Academic Press, London, 2000.

[31] L. Shapiro, G. Stockman, Computer Vision, Prentice Hall PTR, Upper Saddle River, NJ, USA, 2002.

[32] S. Bakke, P.-E. Øren, 3-d pore-scale modelling of sandstones and flow simulations in the pore networks. society of petroleum engineers, Society of Petroleum Engineers 2 (1997) 136 - 149.

[33] P.-E. Øren, S. Bakke, Process based reconstruction of sandstones and prediction of transport properties, Transport in Porous Media 46 (2002) 311-343.

[34] I. Song, J. Renner, Hydromechanical properties of fontainebleau sandstone: Experimental determination and micromechanical modeling, Journal of Geophysical Research: Solid Earth 113 (B9) (2008) n/a-n/a.

[35] C. Voivret, F. Radjaï, J.-Y. Delenne, M. S. El Youssoufi, Spacefilling properties of polydisperse granular media, Phys. Rev. E 76 (2007) 021301. doi:10.1103/PhysRevE.76.021301.

[36] F. Dubois, M. Jean, LMGC90 une plateforme de développement dédiée à la modlisation des problmes d'interaction, Actes du sixiéme colloque national en calcul des structures, CSMAAFM-LMS 1 (2003) 111-118.

[37] F. Dubois, M. Jean, The non smooth contact dynamic method: recent LMGC90 software developments and application, Vol. 27 of Lecture Notes in Applied and Computational Mechanics, Springer Berlin Heidelberg, 2006.

[38] S. C. Cowin, A note on the microstructural dependence of the anisotropic elastic constants of textured materials, in: M. M. C. S. C. Hayley H. Shen, Masao Satake, C. S. Campbell (Eds.), Advances in Micromechanics of Granular MaterialsProceedings of the Second US/Japan Seminar on Micromechanics of Granular Materials Potsdam, NY, USA, August 59, 1991, Vol. 31 of
Studies in Applied Mechanics, Elsevier, 1992, pp. 61 - 70.

[39] T. Kanit, S. Forest, I. Galliet, V. Mounoury, D. Jeulin, Determination of the size of the representative volume element for random composites: statistical and numerical approach, International Journal of Solids and Structures 40 (2003) 3647 - 3679.

[40] M. Bornert, T. Bretheau, P. Gilormini (Eds.), Homognisation en mcanique des matriaux 1 : matriaux alatoires lastiques et milieux priodiques, Hermes Science Publications, Paris, France, 2001.

[41] E. Sanchez-Palencia, Non-Homogeneous Media and Vibration Theory, Springer-Verlag, Berlin, Germany, 1980.

[42] E. J. Garboczi, A. R. Day, An algorithm for computing the effective linear elastic properties of heterogeneous materials: Threedimensional results for composites with equal phase poisson ratios, Journal of the Mechanics and Physics of Solids 43 (1995) $1349-1362$.

[43] A. P. Roberts, E. J. Garboczi, Elastic moduli of model random three-dimensional closed-cell cellular solids, Acta Materialia 49 (2001) $189-197$.

[44] A. P. Roberts, E. J. Garboczi, Elastic properties of model random three-dimensional open-cell solids, Journal of the Mechanics and Physics of Solids 50 (2002) $33-55$.

[45] J.-M. Gatt, Y. Monerie, D. Laux, D. Baron, Elastic behavior of porous ceramics: application to nuclear fuel materials, Journal of Nuclear Materials 336 (2005) 145 - 155.

[46] G. Mavko, T. Mukerji, J. Dvorkin, The Rock Physics Handbook, Cambridge University Press, 2009.

[47] R. Hill, A self-consistent mechanics of composite materials, Journal of the Mechanics and Physics of Solids 13 (4) (1965) $213-222$. 